\title{
Novel Hemizygous Missense Variant of Spermine Synthase (SMS) Gene Causes Snyder-Robinson Syndrome in a Four-Year-Old Boy
}

\author{
Stella Mouskou ${ }^{a} \quad$ Adamantios Katerelos $^{b}$ Artemis Doulgerakic \\ Sofia Leka-Emiri ${ }^{d}$ Emmanouil Manolakos ${ }^{\mathrm{e}}$ loannis Papoulidis $^{\mathrm{e}}$ \\ Athina Ververi ${ }^{\mathrm{e}}$ Georgios Vartzelis ${ }^{\mathrm{a}}$ Anastasia Korona ${ }^{\mathrm{a}}$ Sotiria Mastroyanni ${ }^{\mathrm{a}}$ \\ Konstantinos Voudris ${ }^{a}$ \\ aDepartment of Neurology, 'P \& A Kyriakou' Children's Hospital, Athens, Greece; 'Department of Neurology, \\ 'Karamandaneio' Children's Hospital, Patra, Greece; 'Department of Bone and Mineral Metabolism, Institute of \\ Child Health, Athens, Greece; 'Department of Endocrinology-Growth and Development, 'P \& A Kyriakou' Children's

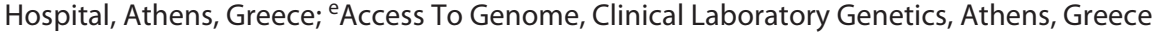

\section{Established Facts}

- Snyder-Robinson syndrome (SRS) is an extremely rare X-linked intellectual disability syndrome.

- The use of whole-exome sequencing in recognizing causes of intellectual disability is established.

\section{Novel Insights}

- Herein, we describe a de novo SMS gene mutation, c.334C>G (p.Pro112Ala), which causes SRS.

- This previously unreported mutation causes a similar phenotype found in cases with SRS.

- The importance of a multidisciplinary approach, with targeted follow-up, comprehensive diagnostic work-up, and proper treatment interventions are highlighted.

\section{Keywords}

Spermine synthase $\cdot$ Snyder-Robinson syndrome $\cdot$ de novo mutation · Osteopenia - Epilepsy

\footnotetext{
Abstract

Snyder-Robinson syndrome (SRS) is an extremely rare $\mathrm{X}$ linked intellectual disability syndrome (MRXSSR; MIM \#309583). The main clinical features of SRS include psychomotor delay, hypotonia, and asthenic-type body habitus -
}

reduced body weight and bone abnormalities (osteoporosis, fractures, kyphoscoliosis). We report a case of SRS with a hemizygous missense variant in the SMS gene,c.334C $>G$ (p.Pro112Ala), in a 4-year-old boy, who initially developed hypotonia, delayed motor skills, and subsequently epilepsy. This variant in SMS was found to be de novo. To the best of our knowledge, this novel SMS gene variant has never been previously reported in disease-related variation databases, such as ClinVar or HGMD.

(c) 2021 S. Karger AG, Base karger@karger.com

(c) 2021 S. Karger AG, Basel

www.karger.com/msy

Karger"
Correspondence to:

Stella Mouskou, stelli_m@yahoo.com 


\section{Introduction}

Snyder-Robinson syndrome (SRS), also known as a deficiency of the spermine synthase syndrome, is an extremely rare X-linked intellectual disability syndrome (MRXSSR, MIM \#309583). It is characterized by hypotonia, asthenic body build with low muscle mass, unsteady gait and severe generalized psychomotor delay with moderate to severe intellectual disability, as well as along, thin, asymmetrical face with prominent lower lip, long fingers and toes, and nasal, dysarthric or absent speech. (PMID: 23696453, GeneReviews NBK144284). Bone abnormalities (e.g., osteoporosis, kyphoscoliosis, fractures, joint contractures) are also characteristic. Myoclonic or myoclonic-like seizures and renal abnormalities have been reported in some patients [Schwartz et al., 2020].

SRS was first described by Dr. Snyder and Robinson in 1969 [Snyder and Robinson, 1969]. It is caused by a genetic defect in the spermine synthase (SMS) gene located in the short arm of the X chromosome (Xp22.11) which was identified by Cason et al. [2003]. The SMS gene (MIM *300105) encodes spermine synthase which converts spermidine into spermine, a polyamine whose deficiency is responsible for the signs and symptoms of SRS [Cason et al., 2003]. Polyamines are essential for normal mammalian cell growth and development. Spermine is an endogenous polyamine with a polycationic structure which is involved in several key cellular processes including proliferation, protein and nucleic acid synthesis, cell adhesion and signaling, and ion channel regulation. Even though the resulting lack of spermine in the cells clearly impacts normal development, including the development of the brain, muscles and bones, it is still unknown how it leads to the specific signs and symptoms of SRS [Murray-Stewart et al., 2018]. A hemizygous pathogenic variant in SMS causes SRS [Cason et al., 2003].

The diagnosis of SRS is confirmed or established based on laboratory testing of the enzymatic activity of the spermine synthase or identification of a hemizygous lossof-function (LoF) pathogenic variant in SMS [Schwartz et al., 2020]. Most published cases are mainly familial.

We present a 4-year-old boy with SRS and a de novo $S M S$ gene mutation, c.334C $>\mathrm{G}$ (p.Pro112Ala), who initially developed hypotonia and delayed motor skills followed by epilepsy. To our knowledge, this variant has not been published in the relevant literature or reported in disease-related variation databases such as ClinVar or HGMD.

\section{Case Presentation}

An 11-month-old male infant was referred for neurological evaluation due to hypotonia and delayed motor skills. He is the first child of nonconsanguineous, healthy parents, born small for gestational age after an uneventful pregnancy. He had no perinatal problems apart from prolonged neonatal jaundice with mild elevation of aminotransferases (ASTmax: $63 \mathrm{mg} / \mathrm{dL}$ and ALTmax: 54 $\mathrm{mg} / \mathrm{dL}$, (normal values: 5-45 $\mathrm{mg} / \mathrm{dL}$ ), which resolved spontaneously. On physical examination, he was a happy infant with good eye contact. Height, weight and head circumference were between the 25th and 50th percentile. Subtle facial dysmorphism was present (long face, small mouth, low-set ears, prominent lower lip). $\mathrm{He}$

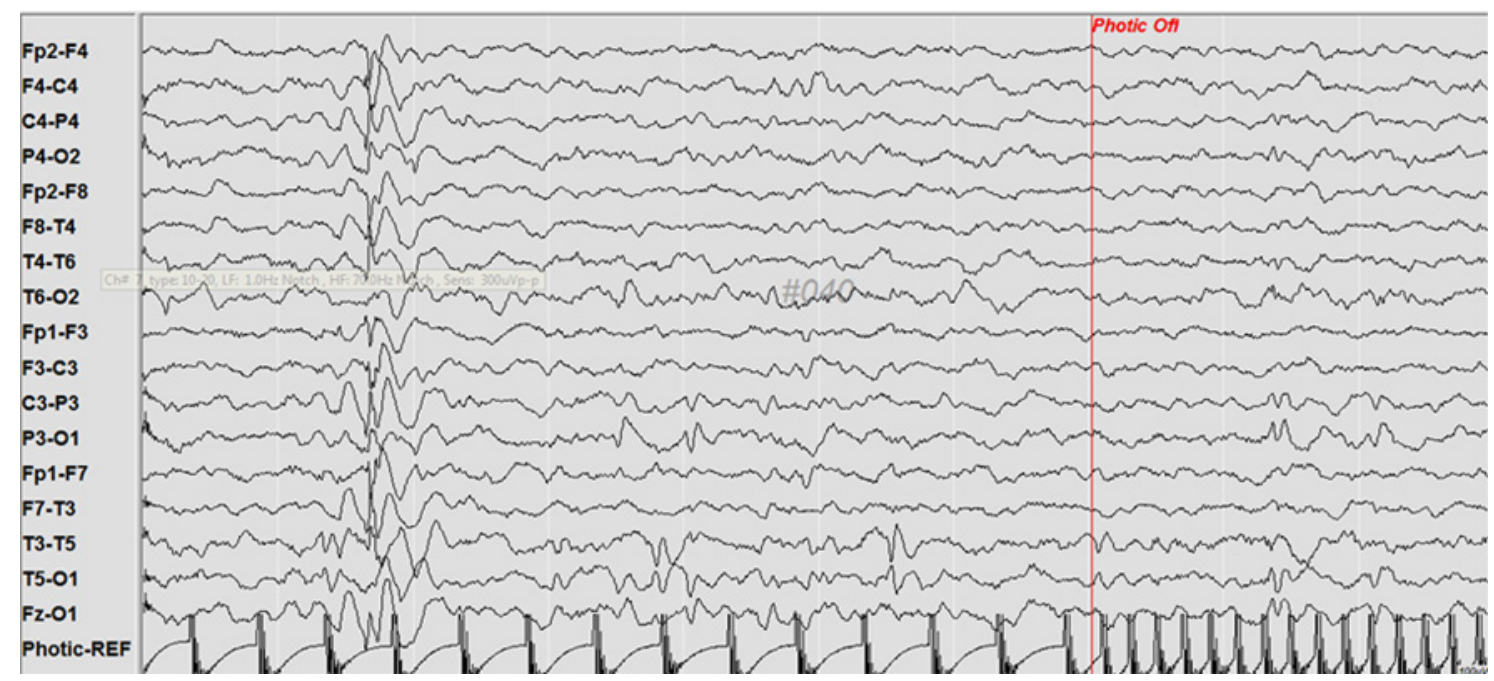

Fig. 1. EEG of the patient at 2 years of age, showing generalized discharges with spike-wave complexes and complexes mainly recorded from left mid-temporal electrodes.

de novo Mutation in SMS Gene Causes

Snyder-Robinson Syndrome
Mol Syndromol 2021;12:194-199

DOI: $10.1159 / 000514122$ 
could sit only with support and had made no attempts to roll over. He had feeding difficulties and central hypotonia. A brain MRI, screening tests for metabolic disorders, karyotyping and molecular analysis for Angelman and Fragile X syndrome did not reveal any pathological findings. An EEG showed bradyarrhythmia without epileptic discharges.

Until the age of 2 years, he received regular clinical care for developmental delay. He walked independently at 18 months of age with physiotherapy support. Despite speech therapy, his active and passive language development remained delayed with echolalia and poor understanding (less than 10 simple words). At that age, he had an episode of loss of consciousness, with gaze fixation and generalized hypotonia. EEG was abnormal with generalized paroxysmal activity, and he was commenced on treatment with valproic acid (Fig. 1). He was free of episodes for almost 10 months, when he presented with similar episodes with trunk instability,

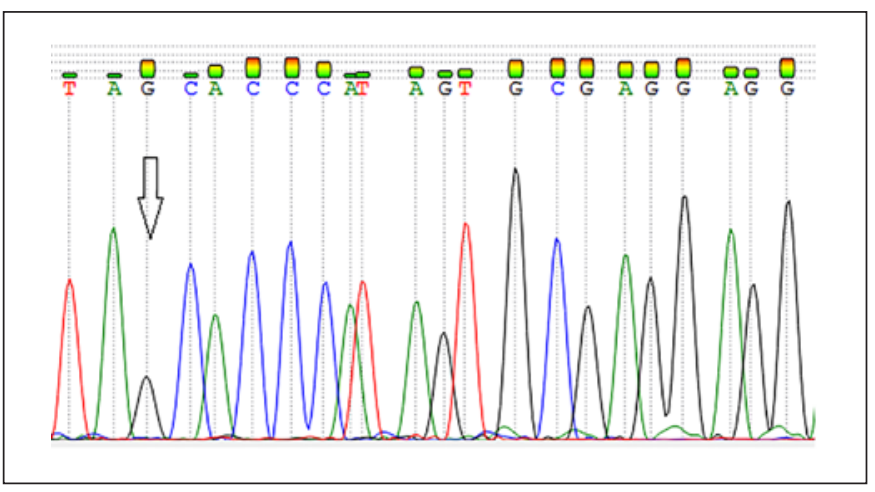

Fig. 2. Nucleotide change c.334C $>$ G (p.Pro112Ala) was identified in hemizygosity in the SMS gene which isassociated with SnyderRobinson syndrome. sticky speech, and episodes of head-drop several times during daytime, which were treated with dosage adjustment.

At the age of 3 years, a significant delay remained in speech as well as gait instability. Because of his dysmorphic features, history of neonatal jaundice and clinical presentation, a test for NiemannPick disease was performed as well as molecular karyotype analysis, and they were both normal. Brain MRI remained without any specific findings. A developmental assessment was also performed, which confirmed a global developmental disorder.

At the age of 4, due to the persistence of seizures, levetiracetam and clobazam were added to his therapeutic regime with partial control of seizures. Due to psychomotor retardation, significant speech delay and epilepsy, the patient underwent further genetic testing by means of next-generation sequencing (NGS) gene panel. Oligonucleotide-based target capture followed by NGS (Illumina NextSeq) were used to capture variants in an epilepsy panel including 283 genes. The software Burrows-Wheeler Aligner (BWAMEM) was used for data analysis (reference genome UCSChg19 and reference database Human Gene Mutation Database [HGMDv.2017.1]). The test was performed on genomic DNA extracted from the patient's peripheral blood. The patient is a carrier of a hemizygous missense variant, c.334C $>\mathrm{G}$ (p.Pro112Ala), in the SMS gene (Fig. 2). Maternal testing was negative, confirming that the the SMS variant had arisen de novo. No other likely pathogenic or pathogenic variants were identified. Another child, a healthy boy, was born before completion of the genetic testing.

At recent reevaluation at the age of $41 / 2$ years, we saw a friendly slender boy with an asthenic body build and low muscle mass. Weight, height and head circumference were all between the 10th and 25th percentile. He showed a good social interaction with peers, had good eye contact, and he was able to follow simple commands. He still had severe global psychomotor delay with severe speech delay (very poor vocabulary, small 2-word sentences), gait instability and hyperextensibility of the elbows. He was on 3 antiepileptic drugs (valproic acid, oxcarbazepine, clobazam), and he was free of seizures. He followed speech, physical, and occupa-

Fig. 3. X-ray of the spine showing diffuse osteopenia without fractures or scoliosis.

Fig. 4. Observer-independent information on cortical thickness and mineralization with digital X-ray radiogrammetry, using BoneXpert ${ }^{\circledR}$ software. The transverse area of the cortical bone is computed, using the cortical thickness, the shaft width and bone length of the three middle metacarpal bones.
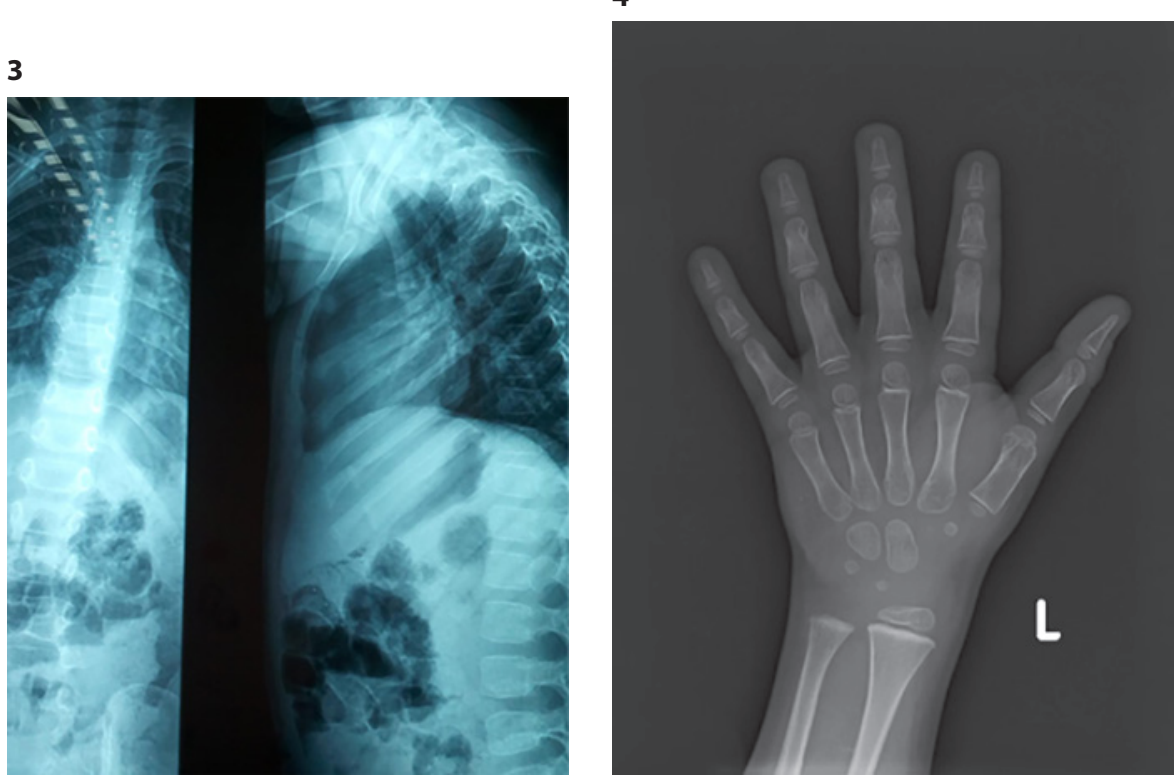
tional therapy on a weekly schedule. A renal ultrasound did not reveal any abnormalities.

In SRS, osteoporosis, which leads to an increased risk of fractures, has been described in early childhood. Our patient was under Levothyroxine and vitamin D treatment since the age of $3 \frac{1}{2}$ years. At the age of 4 , he had one low-energy fracture of the right radius, after a fall from less than standing height. His forearm $\mathrm{X}$-ray revealed osteopenia, slender bones and bone age concordant with his chronological age. Extensive endocrinological testing was normal: blood calcium $=9.9 \mathrm{mg} / \mathrm{dL}(8.8-10.6)$, blood phosphorus $=4.7 \mathrm{mg} / \mathrm{dL}(2.3-4.7)$, blood creatinine $=0.44 \mathrm{mg} / \mathrm{dL}(<0.7)$, urine calcium $/$ creatinine $=0.15 \mathrm{mg} / \mathrm{mg}(0.018-0.39)$, urine phosphorus/ creatine $=1,42 \mathrm{mg} / \mathrm{mg}(0.33-2.19)$, parathyroid hormone $(\mathrm{PTH})$ $=38.8 \mathrm{pg} / \mathrm{mL}(14-88), \mathrm{ALP}=177 \mathrm{U} / \mathrm{lt}(<500), 25-\mathrm{OH}$ vit $\mathrm{D}=29.4$ $\mathrm{ng} / \mathrm{mL}(20-100)$. He did not have kyphoscoliosis. This was confirmed with a baseline X-ray of the spine which showed diffuse osteopenia, but no vertebral deformities suggestive of fractures (Fig. 3).

Due to lack of cooperation, bone mineral density measurement was not possible. Instead, digital X-ray radiogrammetry (DXR) was performed, using a digitalized $\mathrm{X}$-ray of the left hand and a dedicated software (BoneXpert ${ }^{\circledR}$ ) to analyze the image. Apart from the automated bone age assessment, this software offers the option of using the bone health index (BHI) for evaluation of cortical bone thickness, as it reflects the amount of cortical bone in the 3 middle metacarpal shafts (Fig. 4). For proper interpretation, BHI value is expressed as a standard deviation score (SDS) relative to children of the same bone age and gender: a BHI SDS near 0 means that the $\mathrm{BHI}$ is typical of healthy children. As expected, our patient had a very low BHI SDS (-2.6) for his age and gender, thus his osteopenia was confirmed. Six months later, he had a new low-energy fracture of the right arm (Fig. 5).

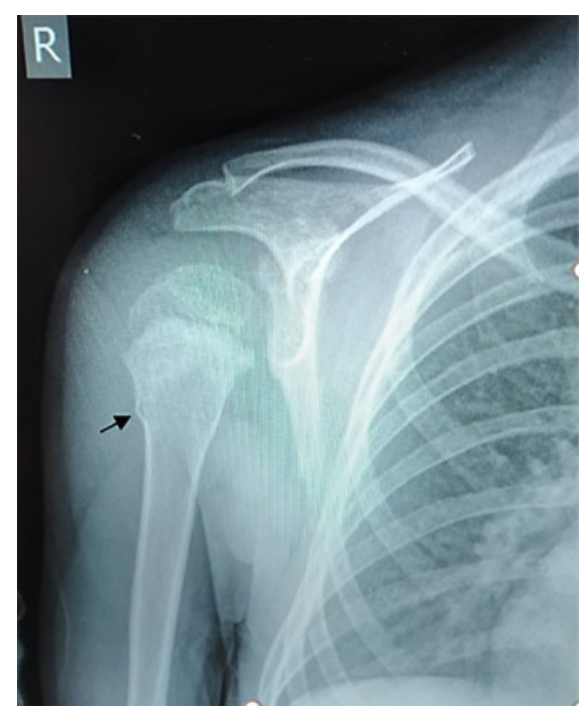

Fig. 5. X-ray of the right arm revealed a low-energy fracture (steplike deformity) at the proximal metaphysis of the R humerus, narrow diaphysis, and osteopenia.

de novo Mutation in SMS Gene Causes

Snyder-Robinson Syndrome

\section{Discussion}

SRS is an X-linked intellectual disability syndrome with a specific clinical phenotype, compatible with our patient's presentation.

Since the gene is located in the X chromosome, only males are affected. According to the most recent literature, 20 individuals from 9 families have been identified with a pathogenic variant in SMS. The 20 reported patients belong to 9 unrelated families. There is wide interand intrafamilial variability. Nevertheless, no genotypephenotype correlation has been described yet [Schwartz et al., 2020]. Of the 20 individuals evaluated and reported in the current literature, 13 were identified in the Americas: Mexico, Brazil, and USA, and 7 were identified in Western European countries [Schwartz et al., 2020]. The prevalence of SRS is unknown [Starks et al., 2018].

The spermine synthase protein consists of 3 domains, the C-terminal, N-terminal, and the central (linker) domain. The index SMS variant is located within the $\mathrm{N}$-terminal, which mediates dimerization, whereas the C-domain carries out the catalysis of spermidine to spermine. Dimerization occurs mainly through interactions between the N-terminal domains [https://www.uniprot. org]. Deletion of the N-terminal domain has been shown to cause complete loss of spermine synthase activity, suggesting that dimerization may be required for activity of the protein [Wu et al., 2008].Pathogenic variants affecting all 3 domains have been reported in the ClinVar database (https://www.ncbi.nlm.nih.gov/clinvar/).

The index variant has not been observed in the large reference population cohorts of the Genome Aggregation Database (gnomAD, $n>120,000$ exomes and >15,000 genomes), https://gnomad.broadinstitute.org/). The p.(Pro$112 \mathrm{Ala})$ residue is highly conserved across species. The variant has not been reported in the literature and is not included in the HGMD Professional or ClinVar databases (https://www.ncbi.nlm.nih.gov/clinvar/). Multiple in silico predictive tools are supportive of a deleterious effect. Another aminoacid change in the same position p.(Pro112Leu) has been previously reported as pathogenic in 2 patients with SRS: 1 in the literature [Peng et al., 2016] and 1 in the Deciphering Developmental Disorders (DDD) study (www.ddduk.org). Based on the classification criteria of the American College of Medical Genetics and Genomics (ACMG), the index patient's variant was classified as likely pathogenic (class 4 ) [Richards et al., 2015].

As mentioned above, the variant had arisen de novo. Of the 20 cases published to date, only 2 individuals were shown to have SRS as the result of a de novo pathogenic

Mol Syndromol 2021;12:194-199 
variant in the SMS gene [Schwartz et al., 2020]. No known carrier mothers have had features of the disease (GeneReviews NBK144284).

Until now, mainly missense variants with a functionally characterized partial LoF have been described. There are 13 disease-causing variants in SMS listed in the HGMD mutation database (October 2019). These include missense variants (85\%) and splicing variants (15\%). Larcher et al. [2020] recently described the first complete LoF variant, Met303Lysfs*, in a male patient with a severe form of SRS, who died at 4 months of age. The authors suggest that pathogenic variants in SMS are indeed LoF, and a genotype-phenotype correlation between the type of variant and the severity of the syndrome may exist [Larcher et al., 2020].

Once an SMS pathogenic variant is identified in an affected family member, carrier testing for at-risk relatives, prenatal testing for pregnancies at increased risk, and preimplantation genetic testing are possible [Schwartz et al., 2020].

During the first decade of life, males with SRS develop osteoporosis, with long bones being most severely affected, as seen in the index patient. Among males reported in the literature so far, osteoporosis and fracture incidence do not progressively worsen with age, but remain at the severity found at the time of diagnosis. The mechanism of decreased bone mineral density is not well understood. To better define the osteoporosis of SRS, Albert et al. [2015] performed detailed studies on affected individuals and provided the first evidence supporting that SRS patients lose osteoblast and osteoclast activity and that the low bone density ocurring in SRS most likely arises by an intrinsic cellular process [Albert et al., 2015]. Furthermore, Ramsay et al. [2019] modeled the disease by silencing SMS in multipotent stromal cells in human bone marrow and found what led to reduced cell proliferation and deficient bone formation in vitro, as evidenced by reduced mineralization and decreased bone sialoprotein expression. This study suggests that SMS deficiency causes strong transcriptomic and metabolic changes in multipotent stromal cells, which are possibly associated with the observed impaired osteogenesis both in vitro and in vivo [Ramsay et al., 2019].

Calcium and/or vitamin D supplementation with regular exercise should be started once low bone mineral density is noted. Calcium supplementation has slightly improved bone mineral density in a few individuals. On the other hand, the use of bisphosphonates is reserved for the most severe cases of osteoporosis and under certain conditions in children [Albert et al., 2015]. Clinical examination, including assessment for kyphoscoliosis and dual-energy x-ray absorptiometry (DXA) scans for diagnosis and follow-up of osteoporosis should be performed on a regular basis. Importantly, when a DXA scan cannot be performed, DXR can be a useful diagnostic adjunct. The relation of BHI SDS to fracture risk is still under investigation; therefore, it is considered a research tool to date. However, preliminary studies show that there is strong, positive correlation between BHI SDS and bone mineral density $Z$ scores, and this is reassuring [Schündeln et al., 2016].

Calcium and vitamin D supplementation should be taken cautiously, in order to avoid ectopic calcifications, e.g., nephrocalcinosis. However, given the dynamic process of skeletal growth and in view of the fact that the index case presents with a new mutation, a periodic orthopedic evaluation is desirable in order to promptly diagnose any skeletal deformity.

Seizures usually occur in early childhood in up to $67 \%$ of the cases reported. Severity and frequency vary considerably. Seizures have shown varying responses to antiseizure medications; carbamazepine, phenobarbital, clobazam, levetiracetam, and valproic acid have been used successfully in some individuals. In our patient, epilepsy is at the time well controlled by 3 antiepileptic drugs. As some of these medications are known to affect bone health, therapeutic decisions should be tailored accordingly, bearing in mind each patient's bone phenotype [Schwartz et al., 2020].

\section{Conclusion}

Herein, we report a 4-year-old boy with SRS carrying a novel SMS gene variant, c.334C > G (p.Pro112Ala), that has not been previously reported in the literature. The clinical findings in our patient are almost identical to the patients reported in the clinical spectrum of SRS. In our opinion, since SRS is extremely rare, molecular testing should be performed in individuals with undefined developmental delay syndrome. It is important that SMS is included in gene panels which are relevant to various phenotypes presenting with, e.g., developmental delay, epilepsy, or osteoporosis. Establishing the diagnosis of SRS in affected individuals is crucial in order to offer a holistic and multidisciplinary approach. This is important for the patients themselves and their families. In addition, the identification of further patients with this extremely rare disorder can shed light on new aspects of the condition, which may ultimately allow for gene-specific and even variant-specific treatment in the future. 


\section{Acknowledgments}

We thank the patient's family for kindly participating in this study and all the physicians that collaborated in diagnosis and clinical follow-up of the patient.

\section{Statement of Ethics}

This case report was produced in accordance with institutional policies; the patient's parents gave written informed consent for publication of this case report and any accompanying photographs.

\section{Conflict of Interest Statement}

The authors have no conflicts of interest to declare.

\section{Funding Sources}

No funding sources to report.

\section{Author Contributions}

All authors made substantive intellectual contributions, read, and approved the final version of the manuscript. S. Mouskou was in charge of overall patient management, preparation, and finalization of the manuscript providing important intellectual inputs. A. Katerelos prepared the initial draft of the manuscript. E. Manolakos, I. Papoulidis, and A. Ververi performed and analyzed the WES data and the analysis of the identified variants. S. Leka participated in the endocrinology follow-up. A.Doulgeraki participated in the skeletal health assessment, including BoneXpert analysis and interpretation. A. Korona, G. Vartzelis, and S. Mastroyanni interpreted clinical data and critically read the manuscript. $\mathrm{K}$. Voudris supervised patient work-up and management.

\section{References}

Albert JS, Bhattacharyya N, Wolfe LA, Bone WP, Maduro V, Accardi J, et al. Impaired osteoblast and osteoclast function characterize the osteoporosis of Snyder-Robinson syndrome. Orphanet J Rare Dis. 2015;10:27.

Cason AL, Ikeguchi Y, Skinner C, Wood TC, Holden KR, Lubs HA, et al. X-linked spermine synthase gene (SMS) defect: the first polyamine deficiency syndrome. Eur J Hum Genet. $2003 ; 11(12): 937-44$.

Larcher L, Norris JW, Lejeune E, Buratti J, Mignot C, Garel C, et al. The complete loss of function of the SMS gene results in a severe form of Snyder-Robinson syndrome. Eur J Med Genet. 2020;63(4):103777.

Murray-Stewart T, Dunworth M, Foley J, Schwartz C, Casero RA Jr. Polyamine Homeostasis in Snyder-Robinson Syndrome. Med Sci (Basel). 2018;6(4):112.
Peng Y, Norris J, Schwartz C, Alexov E. Revealing the Effects of Missense Mutations Causing Snyder-Robinson Syndrome on the Stability and Dimerization of Spermine Synthase. Int J Mol Sci. 2016;17(1).

Ramsay AL, Alonso-Garcia V, Chaboya GC, Rabut B, Le B, Florez J, et al. Modeling Snyder-Robinson Syndrome in multipotent stromal cells reveals impaired mitochondrial function as a potential cause for deficient osteogenesis. Scientific Reports (9) Article number: 15395 (2019).

Richards S, Aziz N, Bale S, Bick D, Das S, GastierFoster J, et al. ACMG Laboratory Quality Assurance Committee. Standards and guidelines for the interpretation of sequence variants: a joint consensus recommendation of the American College of Medical Genetics and Genomics and the Association for Molecular Pathology. Genet Med. 2015;17(5): $405-24$.
Schündeln MM, Marschke L, Bauer JJ, Hauffa PK, Schweiger B, Führer-Sakel D, et al. A Piece of the Puzzle: The Bone Health Index of the BoneXpert Software Reflects Cortical Bone Mineral Density in Pediatric and Adolescent Patients. PLoS One. 2016;11(3):e0151936.

Schwartz CE, Peron A, Kutler MJ. Snyder-Robinson syndrome. 2020.Available from:

Snyder RD, Robinson A. Recessive sex-linked mental retardation in the absence of other recognizable abnormalities: report of a family. Clin Pediatr(Phila). 1969;8(11):669-74.

Starks R, Kirby P, Ciliberto M, Hefti M. SnyderRobinson syndrome. Autops Case Rep. 2018; 8(3):e2018031.

Wu H, Min J, Zeng H, McCloskey DE, Ikeguchi Y, Loppnau P, et al. Crystal structure of human spermine synthase: implications of substrate binding and catalytic mechanism. J Biol Chem. 2008;283(23):16135-46. 